\title{
IVD.06 - Closing the gap in early diagnosis of autoimmune rheumatic diseases: discovery of novel biomarkers using high-density peptide microarrays
}

\author{
Kirsten Heiss ${ }^{1}$; Pedro S. R. Simonini ${ }^{1 *}$; Renate Sekul ${ }^{1}$; Volker Stadler ${ }^{1}$. \\ 1PEPperPRINT GmbH, Heidelberg, Germany.
}

Introduction: Autoimmune rheumatic diseases such as rheumatoid arthritis (RA) and psoriatic arthritis (PsA) are attributed to inflammation affecting the joints and connective tissues, with extra-articular manifestations in some disease types. Early diagnosis is the key to optimal therapeutic success in order to slow down disease progression or even prevent joint damage and hence irreversible disability. However, diagnosis in an early disease stage is often challenging. In RA, up to $30 \%$ of early stage patients are negative for the current serological diagnostic measures using antibodies against cyclic citrullinated peptide antigens (ACPA) or rheumatoid factor (RF). In PsA, both RF and ACPA are mainly absent. Moreover, the heterogeneous disease phenotypes associated with PsA often make an early diagnosis difficult, especially in patients without psoriatic skin lesions.

Objective: The goal of this study is to perform a comprehensive analysis of antigenic proteins and their underlying epitopes in order to identify novel biomarkers for RA and PsA and develop innovative serological tests with a higher sensitivity for early disease diagnosis.

Methodology: High-density peptide microarrays are a great tool to screen large libraries of peptides against serum antibodies. We build a library of $>100.000$ linear and conformational peptides from selected known and candidate disease-associated autoantigens. The library further included peptides containing posttranslational modifications such as citrullination and carbamylation to allow simultaneous comparison of antibody response to tens of thousands native and their corresponding modified peptides. We then screened sera from different disease cohorts (and disease stages) and healthy controls against the library in order to identify novel biomarkers in PsA and RA.

Results: In a first screening we analyzed clinically-defined sera from 4 cohorts (control, PsA, early-stage seronegative RA and late-stage seropositive RA) against a highly diverse peptide library covering $>100.000$ peptides. We identified $>5.000$ statistically significant peptides which were further validated in a second independent screening. These peptides built clusters showing a higher prevalence in their respective disease cohorts, particularly in early disease stages, allowing a clear differentiation between disease and control cohorts. We further identified novel epitopes in previously seronegative early-stage RA patients, as well as unique epitopes associated with PsA. These peptides are potential new biomarkers for the diagnosis of early RA and PsA. However, further validation is required.

Conclusion: Differentiation on epitope level but not on protein level yielded a more comprehensive picture of antigenic motifs specifically recognized in autoimmune rheumatic diseases. These autoantibodies can potentially serve as biomarkers for early diagnosis of rheumatoid arthritis and psoriatic arthritis. Beyond that, our approach may also help to identify markers for patient stratification for the treatment of autoimmune rheumatic diseases as there is an urgent need for the discovery of reliable biomarkers predicting the response of patients to respective treatments.

Keywords: Peptide microarray; Biomarker discovery; Autoimmune disease 